\title{
Experimental Evidence of Fine Structure in Channeling Lines
}

\author{
H. O. Lutz, R. Ambros, C. Mayer-Böricke, K. Reichelt, and M. Rogge \\ Kernforschungsanlage Jülich, Germany \\ (Z. Naturforsch. 26 a, 1105-1108 [1971] ; received 20 April 1971)
}

\begin{abstract}
Fine structure was found in the angular distribution of $2 \mathrm{MeV} \mathrm{He}$ ions channeled between the (001) planes of 1600 to $3000 \AA$ thick Au single crystals. In addition, the energy loss of the channeled particles was determined as a function of exit angle from the crystal.
\end{abstract}

\section{Introduction}

The path of energetic ions channeled between densely packed lattice planes of a single crystal is governed by correlated small angle collisions. This results in well defined oscillatory particle paths in planar channels ${ }^{1}$. The angular distribution of these particles after emergence from the crystal is intensely peaked in the forward direction. As has been pointed out ${ }^{2}$, this angular distribution exhibits fine structure which is caused by the correlation between the coordinates of ion entrance into the channel and the exit angle from the channel. In very thin crystals (channel length $l$ only a few oscillation wavelengths $\lambda$ ) this structure is mainly determined by the elastic potential interaction between the ion and the lattice atoms. A detailed study of the angular distribution should therefore give information on the potential parameters involved without simultaneously having to imply an a-priori energy loss function ${ }^{3,4}$.

In the work reported here, the angular distributions of $2 \mathrm{MeV}$ He-ions channeled between the (001) planes of 1600 to $3000 \AA$ thick Au single crystals were studied systematically. The energy loss of the channeled particles has also been determined.

\section{Experimental}

The critical acceptance angle $\alpha_{\mathrm{c}}$ of a low indexed planar channel for light ions of $\mathrm{MeV}$ energies is generally about $1^{\circ}$ or less. If structure in the angular distribution of channeled particles is to be resolved, the angular detector resolution as well as the perfection of crystal orientation ("mosaic spread") has to be at

1 S. Datz, C. Erginsoy, G. Leibfried, and H. O. Lutz, Ann. Rev. Nucl. Sci. 17, 129 [1967].

2 M. Rogge, W. DünNWEber, and C. MaYer-Böricke, Z. Naturforsch. 24 a, 660 [1969].

3 H. O. Lutz, S. Datz, C. D. Moak, and T. S. Noggle, Phys. Rev. Lett. 17, 285 [1966]. least one order of magnitude better. $\mathrm{Ge}$ and $\mathrm{Si}$ single crystals, etched down to less than $1 \mu$ thickness, have sufficiently small mosaic spread. However, it was found that they are very susceptible to radiation damage which constitutes a great experimental difficulty. Therefore, the hetero-epitaxial growth of Au single crystals on mica was studied in detail ${ }^{5}$; Au crystals with high perfection and small mosaic spread $\left(\sim 0.06^{\circ}\right)$ could be obtained recently. Their surface normal was parallel to a 111-direction.

In the present experiments, a well collimated beam $\left(0.002^{\circ}\right)$ of $2 \mathrm{MeV} \mathrm{He}^{+}$-ions was incident on a $\mathrm{Au}$ single crystal of $1300 \AA( \pm 10 \%)$ thickness. The angle between the surface normal and the beam direction was about $35.2^{\circ}$, the incidence angle to the (001) plane $\left|a_{0}\right|<\left|a_{\mathrm{c}}\right|$. The crystal was mounted on a goniometer which permitted rotation about three orthogonal axes. The effective crystal thickness could be varied between $1600 \AA$ and $3000 \AA$ by tilting it about $\langle 001\rangle$ without changing $\alpha_{0}$. The mosaic spread was approximately $0.1^{\circ}$.

The emergent ions were detected in a position sensitive solid state detector ${ }^{6}$ at a distance of $1.38 \mathrm{~m}$ from the crystal. This device subtended a total angle of $2^{\circ}$, sufficiently large to detect the entire channeling angular distribution in one spectrum. A small on-line analogue computer was used to eliminate the energy response of the detector by dividing the $(E x)$-pulse by the $E$-pulse. The angular resolution thus obtained was approximately $0.07^{\circ}$.

In addition, the energy spectra for several crystal lengths were measured in steps of ca. $0.04^{\circ}$ with a silicon surface barrier detector of high energy resolution. An aperture was placed in front of this detector, giving an angular resolution of $0.02^{\circ}$. The system energy resolution was $15 \mathrm{keV}$. The spectra thus obtained as a function of exit angle, $\alpha_{e}$, were normalized in intensity by using the angular distributions measured with the position sensitive detector.

4 S. Datz, C. D. Moak, T. S. Noggle, B. R. Appleton, and H. O. Lutz, Phys. Rev. 179, 315 [1969].

5 K. Reichelt and H. O. Lutz, to be published in J. Crystal Growth.

6 Nuclear Diodes, Inc., Chicago, Ill. 


\section{Results and Discussion}

Figure 1 a shows the variation of the angular distribution with crystal length. The angle of incidence with respect to the (001) planar channel, $\alpha_{0}=-0.17^{\circ}$, remains constant for all distributions. The angles are given with respect to the channel mid-plane. $\left|\alpha_{0}\right|$ is smaller than $\left|\alpha_{\mathrm{c}}\right|$, the critical acceptance angle. $\alpha_{\mathrm{c}}$ was measured by displacing the position sensitive detector $2^{\circ}$ from the primary beam direction across the blocking shadow of the (001) channel. $\alpha_{\mathrm{c}}$ was found to be $0.55^{\circ}$ (full width half minimum), or about $0.9^{\circ}$ (full width full minimum, i. e., the angular distance between the blocking shoulders). The different angular distributions are normalized to the total number of counts in every distribution. Directional channels have been omitted.

The distributions show pronounced structure which changes rapidly with crystal thickness, $l$. Several peaks (up to three) appear in one spectrum. These peaks change in position as the crystal thickness is increased. Simultaneously, their intensities change. At approximately the direction of the in-

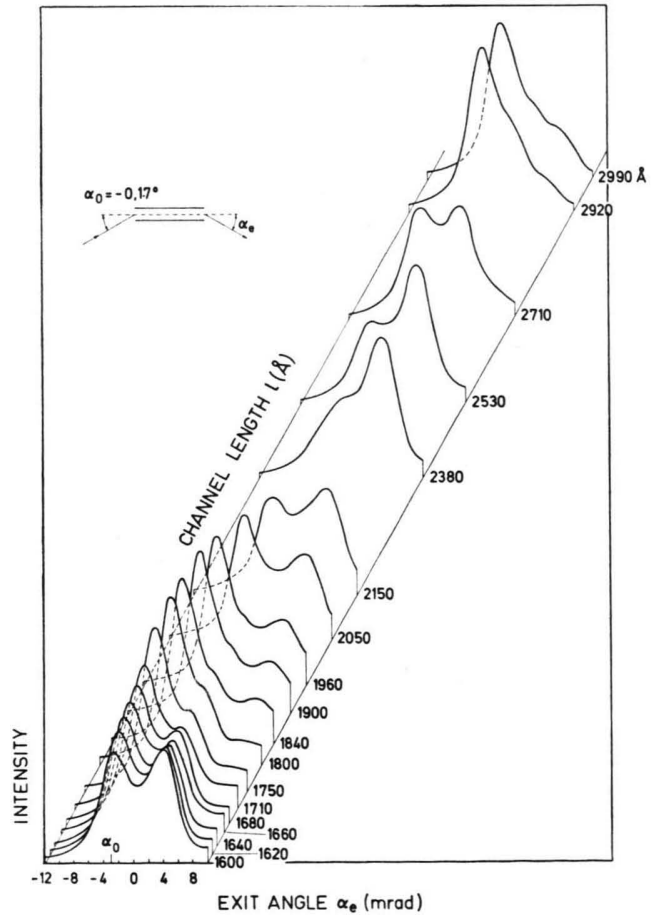

cident beam $\left(\alpha_{\mathrm{e}} \sim \alpha_{0}\right)$, a peak appears in all spectra. Its positions varies less rapidly with $l$.

Assuming a rigorous correlation between the coordinate of entrance into the channel, $y_{0}$, and the angle of exit from the channel, $\alpha_{\mathrm{e}}$, the intensity $\mathrm{d} N / \mathrm{d} \alpha_{\mathrm{e}}$ is proportional to $\mathrm{d} y_{0} / \mathrm{d} \alpha_{\mathrm{e}}$. Extrema in the intensity distribution may, therefore, be expected for angles where $\mathrm{d} \alpha_{\mathrm{e}} / \mathrm{d} y_{0}=0$, or $\mathrm{d}^{2} y_{0} / \mathrm{d} \alpha_{\mathrm{e}}^{2}=0$, respectively. A special case is a trough potential giving rise to a zig-zag-motion. Except at $l \approx n d / \alpha_{0}$, there will always be two maxima (at $-\alpha_{0}$ and $+\alpha_{0}$ ) for each channel length $l$. $d$ is the channel width, $n=1,2,3, \ldots$. Mosaic spread of the crystal will give rise to a broadening of the peak at $\alpha_{\mathrm{e}}=-\alpha_{0}$, the specular entrance angle, and a shift of its position.

To put the discussion on a more quantitative basis, we studied the channeled ion trajectories in greater detail. The planar potential employed was $\Phi(y)=A \cosh (y / c)$. This potential has been used and discussed extensively in previous analyses of channeling trajectories ${ }^{4,7}$. The influence of the in-

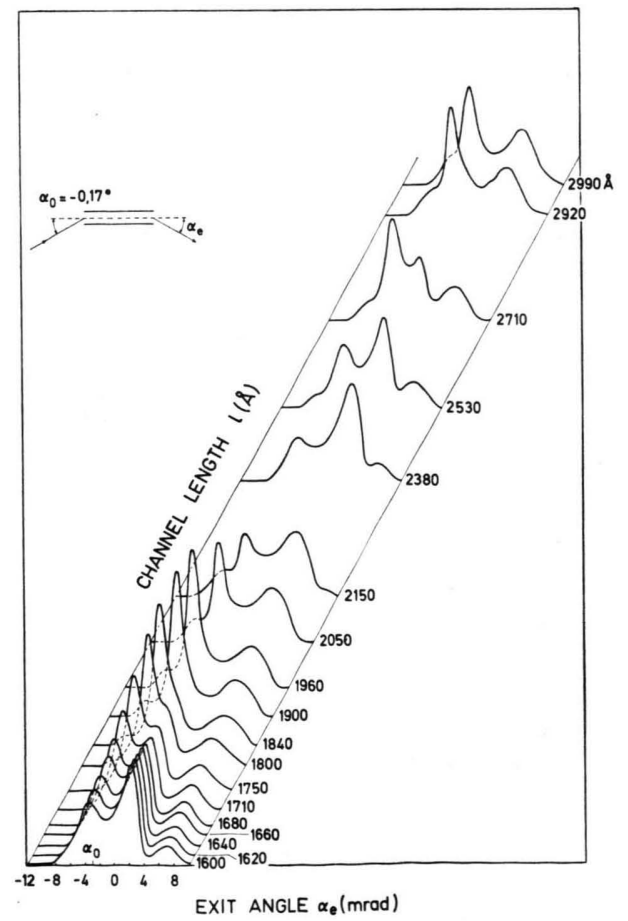

Fig. 1. Angular distributions of He-ions emerging from Au(001)-planar channels as a function of channel length, $l$. Incident energy $2 \mathrm{MeV}$, angle of incidence $a_{0}=-3 \mathrm{mrad}\left(-0.17^{\circ}\right)$. a) Experimental; b) calculated with $A=11 \mathrm{eV}$, $c=0.3 \AA, f=2 \operatorname{mrad}\left(0.11^{\circ}\right)$.

7 M. T. R )BInson, Phys. Rev. 179, 327 [1969]. 
elastic energy loss on the trajectory was not taken into account since the path length was fairly short, $l \lesssim 3 \lambda$. The angle of exit, $\alpha_{\mathrm{e}}$, was calculated numerically for each entrance coordinate, $y_{0}$. The entrance coordinate, $y_{0}$, was varied step by step to simulate a large number of particles penetrating the crystal (up to 4000). The mosaic spread of the crystal was introduced into the calculation by varying $\alpha_{0}$ and $\alpha_{\mathrm{e}}$ accordingly. The probability distribution of the mosaic spread was assumed to be of Gaussian shape with $f$ the FWHM. The finite experimental angular resolution was simulated by smoothing the calculated angular distributions three times over 5 channels ( 5 channels correspond to $1 \mathrm{mrad}$ ).

Figure 2 shows the dependence of $\alpha_{\mathrm{e}}$ on $y_{0}$ for $A=11 \mathrm{eV}, c=0.3 \AA, \alpha_{0}=-0.17^{\circ}, l=2530 \AA, E$ $=2 \mathrm{MeV}, \mathrm{Au}$ planar (001) channel. Several maxima in the intensity distribution occur (upper part of Fig. 2, 2000 trajectories). The results obtained using a finite mosaic spread, $f=0.11^{\circ}$, is indicated by the dashed curve (4000 trajectories). The parameters $A$ and $c$ used in Fig. 2 give a good overall fit between the experimental (Fig. 1 a) and theoretical (Fig. $1 \mathrm{~b})$ angular distributions. They are identical with the parameters derived in Refs. ${ }^{4,7}$ for $3 \mathrm{MeV} \mathrm{He}$ ions in $\mathrm{Au}$ (001) planar channels. Evidently, the intensity distributions are influenced by the mosaic structure of the crystal. This limits the accuracy of the fitting parameters $A$ and $c$, e. g., a comparable fit could be obtained using $A=15 \mathrm{eV}, c=0.34 \AA$, and $f=0.14^{\circ}$.

The accuracy of $A$ and $c$ is firstly determined by the accuracy with which the channel length is known. Furthermore, because of ambiguities in $A-c$ com. binations it seems necessary to fit not only one strong peak (for example the one caused by particles which enter the channel in the vicinity of the midplane) but the entire angular distributions including the lowly populated groups, in order to get high accuracy in an independent determination of the parameters $A$ and $c$. In this case, the mosaic spread of the crystal must be negligibly small $\left(f \lesssim 0.01^{\circ}\right)$, which still appears to be quite difficult to achieve.

The experimentally determined energy loss spectra as a function of $\alpha_{\mathrm{e}}$ are shown for a typical channel length $l(2530 \AA)$ in Figure 3. At $\alpha_{\mathrm{e}}=-0.6^{\circ}$ ( $-10.4 \mathrm{mrad})$, one relatively wide peak shows up. Increasing $\alpha_{\mathrm{e}}$, this peak is shifted slightly in energy and becomes narrower. Within a small angular

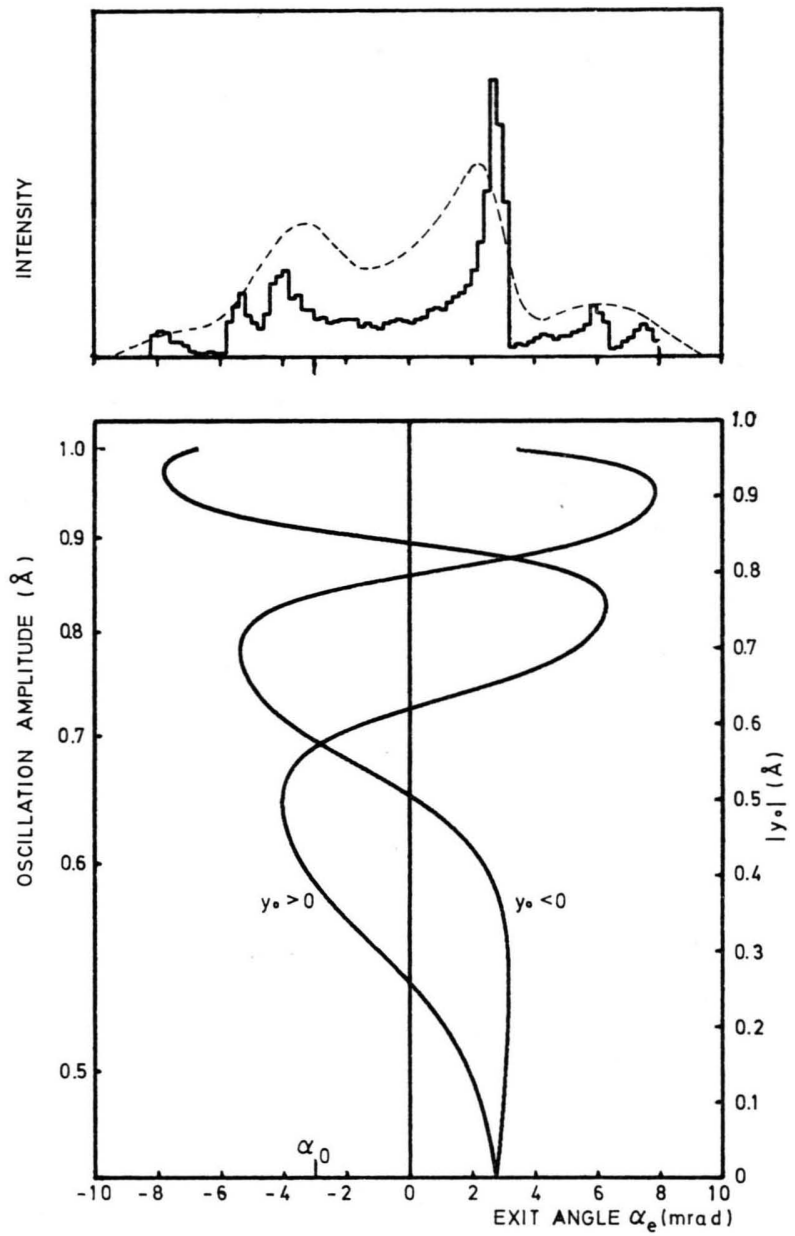

Fig. 2. Calculated dependence of the exit angle $\alpha_{\mathrm{e}}$ on the channel entrance coordinate, $y_{0} . l=2530, A=11 \mathrm{eV}, c=0.3 \AA$. The resulting angular distribution is shown in the upper part of the figure. - no mosaic spread in the crystal, --- mosaic spread $f=2 \operatorname{mrad}\left(0.11^{\circ}\right)$.

range it tends to disappear, while a new peak is coming up. Increasing $\alpha_{\mathrm{e}}$ further, this process is reversed. This behavior can be explained with the help of Figure 2. At large $\left|\alpha_{\mathrm{e}}\right|$, only trajectories with large $\left|y_{0}\right|$ (large energy loss) contribute. Decreasing $\left|\alpha_{\mathrm{e}}\right|$, a new set of trajectories having low energy loss (oscillation amplitude ca. $0.5 \AA$ ) will be detected in the region of $-\alpha_{0}$. This group is very intense (large $\mathrm{d} y_{0} / \mathrm{d} \alpha_{\mathrm{e}}$ and small $\mathrm{d}^{2} y_{0} / \mathrm{d} \alpha_{\mathrm{e}}^{2}$ ).

Since $l \lesssim 3 \lambda$, only few separate energy loss groups can be detected simultaneously at a given $\alpha_{\mathrm{e}}$. Except at certain $\alpha_{\mathrm{e}}$, they are populated quite differently. Higher energy loss groups can be seen only at large $\left|\alpha_{\mathrm{e}}\right|$ where the intense low loss group 


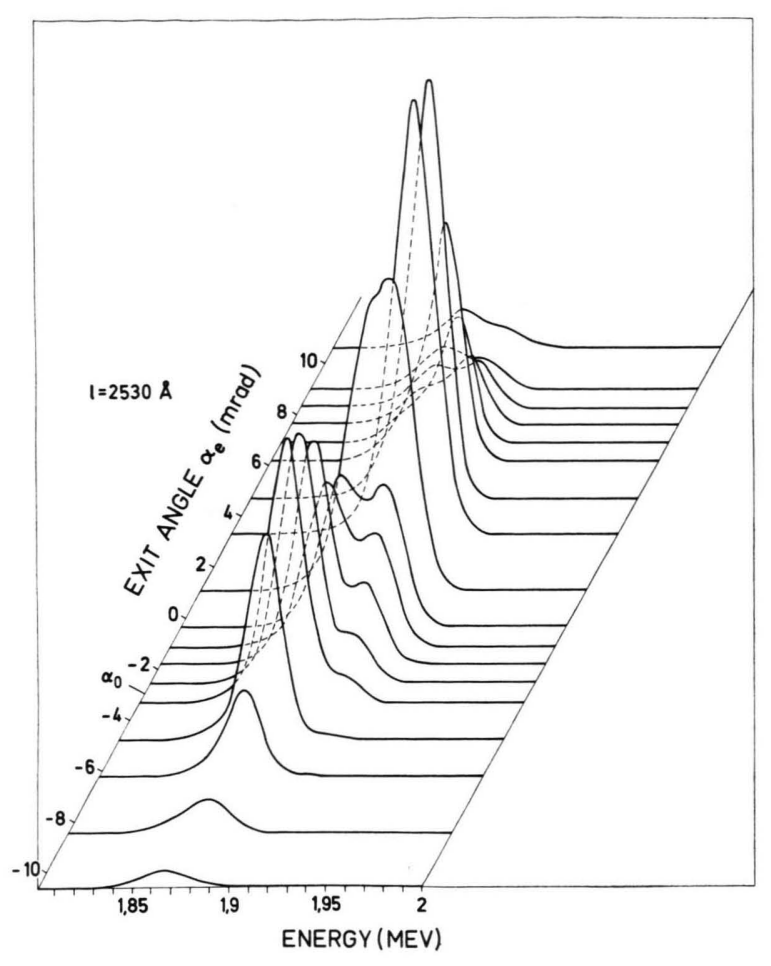

Fig. 3. Experimentally determined energy spectra of He-ions emerging from a $\mathrm{Au}(001)$-planar channel. Incident energy $2 \mathrm{MeV}, a_{0}=-3 \operatorname{mrad}\left(0.17^{\circ}\right), l=2530 \AA$. cannot occur. At small $\left|\alpha_{\mathrm{e}}\right|$, the latter predominates. This would facilitate the mapping of trajectories as suggested previously ${ }^{4}$ : The energy loss may be used to tag a discrete oscillation group in the channel; changing the channel length in small steps, the tag. ged oscillation group can then be found again for each $l$ at a different $\alpha_{\mathrm{e}}$. In the work reported here, the mosaic spread of the crystals was still too large to apply this method with good accuracy.

The methods discussed above are supplementary to previous analyses of channeled ion trajectories. Crystals with small mosaic structure have to be used. The distinct advantage is that only the interaction potential enters the calculations. The energy loss function can in principle be obtained without presuming an a-priori impact parameter dependence.

\section{Acknowledgment}

We are indebted to Prof. H. Schopper, Institut für Kernphysik, University of Frankfurt, for making available to us the $2 \mathrm{MeV}$ van de Graaff accelerator facility. We are particularly grateful to Dr. R. BAss, K. MeINEL, and K. SCHNEIDER for valuable assistance. 